\title{
State Space Models for Power SiC MOSFET
}

\author{
N. K. M'Sirdi, K. Frifita, E. Baghaz, A. Naamane, and M. Boussak \\ Aix Marseille Univ, Universite de Toulon, CNRS, LIS, Marseille, France \\ SASV group of LIS UMR CNRS 7020, 13397, Marseille France, e-mail: \\ nacer.msirdi@univ-amu.fr
}

\begin{abstract}
This paper proposes a State Space Model for a power a Silicon Carbide ( $\mathrm{SiC}$ ) MOSFET. The model uses the electrical EKV MOSFET structure. The model is developed for the SiC MOSFET C2M0025120D CREE (1200V, 90A) and uses the parameters extracted from datasheet
\end{abstract}

Keywords: SiC MOSFET State Space Model, State Space Simulation Model.

\section{Introduction}

In high power applications, Silicon $(\mathrm{Si})$ circuits are more and more replaced by $\mathrm{SiC}$ MOSFET devices. The wide range of operation lead to parameter variations, inherent perturbations and constraints which affect behavior of the components. Then disposal of good simulation models are necessary to get realistic simulations and accurate results.

The increasing use of switching devices in converters in power systems give more abilities but results in complex control problems related to nonlinearities involved and distortion in the electric network. Non robust controls result in a degradation of the power factor and generate voltage and current disturbances which have a different frequency than the fundamental. The state space models are helpful for systems analysis advanced controllers design. Up to now and to the best of our knowledge, the only author talking about state space representation for a MOSFET is Karvonen [1,2]. The model used, is based on SPICE® equations of discrete elements whose values are extracted from datasheet. But only the state vector is presented in the paper and in the thesis. The author uses the Modified Nodal Analysis (MNA) [3,4]. The state space equation have never been developed for MOSFETs and proposed in literature.

In this paper, we propose state space representations, for a SiC MOSFET, deduced from a circuit model we have recently proposed $[5,6]$, based on the well known EKV MOSFET model [7]. The model is developed for the SiC MOSFET transistor C2M0025120D CREE (1200V, 90A) [8] and is implemented in Matlab/Simulink simulation softwares to allow easy control design for $\mathrm{SiC}$ MOSFET based applications. The results show that the proposed model is the most interesting to develop advanced controllers, compared to all the literature existing models. 


\section{SiC MOSFET Model}

\subsection{The Modelling Context}

The MOSFET Models Several simulators have been proposed to describe the device conception and its behaviour. In [9], we find for an electro thermal simulator integrated into the environment. This is based on the use of the Verilog-A description language and the SKILL script language. The VERILOG-A language is used for modeling electrical components and for thermal modeling. Circuit design does not require all details which make such models very slow to run. The EKV model is an accurate mathematical MOSFET model developed by C. C. Enz, F. Krummenacher and E. A. Vittoz based in the 1980s, which takes into account physical properties [7].

The manufacturers propose as much as possibles models for their new circuits. Recently CREE proposed an LTspice simplified models for SiC MOSFETs like the one we are interested in [8]. The models proposed in Psim, Pspice, LMS AMESIM, Saber simulation softwares are often redundant, need solvers and complex. Linearized models are valid only in a restricted area. In order to get credible results based on simulations, we need to have accurate models, with acceptable precision.

In [1]the proposed MOSFET model is base on combined nodal and voltage loops analysis to get the equivalent circuit equations (by applying Kirchhoff's laws) to evaluate the states and their derivatives [10]. The main contribution of [2] is the Gate-Drain capacitance characterization and its dependance on the current flow.

\subsection{The nominal Electric Model}

The electrical model of a SiC MOSFET proposed in [5] is based on the EKV's one. The electric equivalent circuit of figure 1 summarizes the model description like it has been used in physical modeling based simulation softwares like Saber, Pspice and Psim. The Source, Gate and Drain voltages are respectively $V_{S}, V_{G}$ et $V_{D}$. The resistance $R_{G}$ estimates the gate losses as well as $R_{D}$ and $R_{S}$ are used to account for losses at the Source and Drain electrodes. The circuit includes also 3 inductors in series with the electrodes $\left(L_{G}, L_{D}, L_{S}\right)$ to describe the behavior at high frequencies.

In this model, we used parameters extracted from the data-sheet $[5,6]$. The model accounts for a MOSFET operation in 3 regions. It is composed by two current sources $I_{G 1}$ and $I_{G 2}$. For interpolation between these 3 regions of operation, a linear combination of logarithmic functions is used by EKV. The specific current is $I_{s_{0}}=2 \cdot V_{t h}^{2} \cdot K_{s} \cdot g_{m}$. The Drain-Source current $\left(I_{D S}\right)$, in the EKV MOSFET model, is defined by equation (??), as the difference between the direct $I_{G 1}(2)$ and the reverse one $I_{G 2}(3)[6]$. 


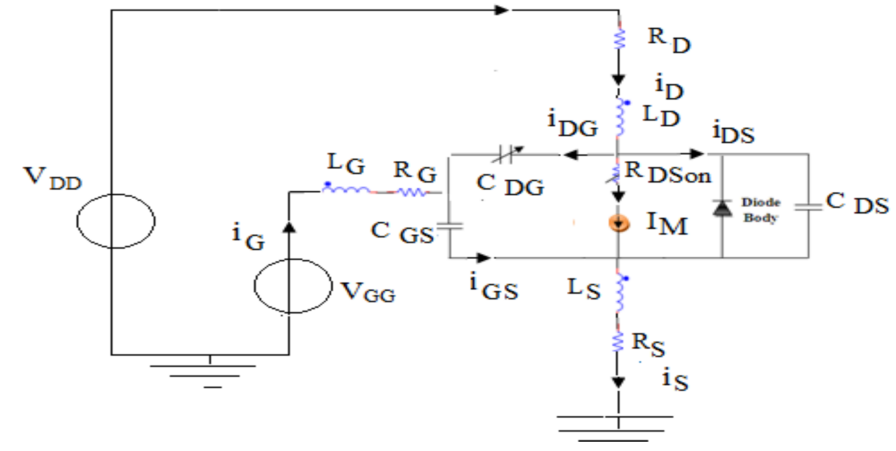

Fig. 1. Electric model of the SiC MOSFET (C2M0080120D)

$$
\begin{aligned}
& I_{M}=I_{G 1}\left(V_{p}-V_{S}\right)-I_{G 2}\left(V_{p}-V_{D}\right) \\
& I_{G 1}=I_{s} \cdot g_{m} \cdot\left[\operatorname{Ln}\left(1+\exp \frac{V_{p}-V_{s}}{2 V_{D S}}\right)\right]^{2} \\
& I_{G 2}=I_{s} . f t \cdot\left[\operatorname{Ln}\left(1-\exp \frac{V_{p}-V_{D}}{2}\right)\right]^{2}
\end{aligned}
$$

$g_{m}$ is the transconductance, $K_{s}$ is the slope factor, $V_{p}=\frac{\left(V_{G}-V_{t h}\right)}{K_{s}}$ the pinch voltage with $V_{t h}$ the thermodynamic reference voltage and $f t$ the internal thermal voltage.

The following electric equations corresponding to the figure (1) can then be obtained applying the Kirchhoff laws for each current node and voltage loop of the circuit. The equation system is redundant and non linear. Let us note $\xi \epsilon\{0,1\}$ a state control variable introduced to switch betokening the ON/OFF states. We have $\xi=1$ when the MOSFET is in ON state and respectively $\xi=0$ when the MOSFET is in the OFF state.

$$
\left\{\begin{array}{c}
V_{G G}=L_{G} \frac{d i_{G}}{d t}+R_{G} i_{G}+V_{G S}+V_{S} \\
V_{D D}=L_{D} \frac{d i_{D}}{d t}+R_{D} i_{D}+V_{D S}+V_{S} \\
V_{D D}-V_{G G}=L_{D} \frac{d i_{D}}{d t}+R_{D} i_{D}+V_{D G}-L_{G} \frac{d i_{G}}{d t}-R_{G} i_{G} \\
V_{S}=L_{S} \frac{d i_{S}}{d t}+R_{S} i_{S} \\
V_{D S}=\xi \cdot R_{D S o n} I_{M} \\
i_{D S}=C_{D S} \frac{d V_{D S}}{d t} \\
i_{G S}=C_{G S} \frac{d V_{G S}}{d t} \\
i_{D G}=C_{D G} \frac{d V_{D G}}{d t} \\
i_{G}=C_{G S} \frac{d V_{G S}}{d t}-C_{D G} \frac{d V_{D G}}{d t} \\
i_{D}=\xi \cdot I_{M}+C_{D G} \frac{d V_{D G}}{d t}+C_{D S} \frac{d V_{D S}}{d t} \\
i_{S}=\xi \cdot I_{M}+C_{G S} \frac{d V_{G S}}{d t}+C_{D S} \frac{d V_{D S}}{d t}
\end{array}\right.
$$

The Modified Nodal Analysis (MNA) is in general used to solve this set of simultaneous, redundant and non linear equations $[3,4]$ by selecting appropriate variables. The best formulation, to avoid redundancy, computation burden and 
singularities, for simulation and the control design, is the state space form, after simplifying the model.

The inputs are the voltage sources $\left(u(t)=\left[V_{G G}, V_{D D}\right]^{T}\right)$ and the outputs may be all the state variables $\left(i_{G}, i_{D}, i_{S}, V_{G S}, V_{D G}, V_{D S}\right)$ or only some of them as needed by the application considered.

\subsection{State Space SiC MOSFET Model}

The SiC MOSFET inputs are the two voltage sources $\left(V_{D D}, V_{G G}\right)$. The MOSFET model is in general implemented as an electric circuit (see Fig. 1) completed with an appropriate elements to switch between $\mathrm{ON}$ and $\mathrm{OFF}$ states and introducing the non linear current of equation (??) [1]. The system of equation (4) deduced by application of Kirchoff's laws is redudant and has different time and values scales. The input, the outputs and the state equation have to be carefully selected.

First State Space formulation trial The SiC MOSFET circuit is made of six energy storage elements (3 capacitances and 3 inductances). Storage elements are obviated in the system equations (4). The corresponding energy storage variables are, in nature, the internal system states (to be used for the state space representation).

We can keep as state variables the 3 currents (charging the capacitances) and the 3 voltages (inductors), which seem, a priori, independant. The chosen state vector $X$ is then composed by $v_{1}$ and $v_{2}$.

$$
\begin{aligned}
& v_{1}=\left[\begin{array}{lll}
i_{G} & i_{D} & i_{S}
\end{array}\right]^{T} \text { and } v_{2}=\left[\begin{array}{lll}
V_{G S} & V_{D G} V_{D S}
\end{array}\right]^{T} \\
& X=\left[\begin{array}{lllll}
i_{G} & i_{D} & i_{S} & V_{G S} & V_{D G} \\
V_{D S}
\end{array}\right]^{T}
\end{aligned}
$$

Let us keep only a minimum of independant equations (3 for voltages Differential Equations (DAE) and 3 for currents ones):

$$
\begin{gathered}
\left\{\begin{array}{c}
L_{G} \frac{d i_{G}}{d t}+L_{S} \frac{d i_{S}}{d t}+V_{G S}+R_{G} i_{G}+R_{S} i_{S}=V_{G G} \\
L_{D} \frac{d i_{D}}{d t}-L_{G} \frac{d i_{G}}{d t}-R_{G} i_{G}+R_{D} i_{D}+\xi \cdot R_{D S O n} I_{M}-V_{G S}=V_{D D}-V_{G G} \\
\frac{d i_{S}}{d t}=\frac{d i_{D}}{d t}+\frac{d i_{G}}{d t}
\end{array}\right. \\
\left\{\begin{array}{c}
-C_{D G} \frac{d V_{D G}}{d t}+C_{G S} \frac{d V_{G S}}{d t}=i_{G} \\
C_{D S} \frac{d V_{D S}}{d t}+C_{G S} \frac{d V_{G S}}{d t}=i_{S}-\xi \cdot I_{M} \\
\frac{d V_{D S}}{d t}-\frac{d V_{D G}}{d t}-\frac{d V_{G S}}{d t}=0
\end{array}\right.
\end{gathered}
$$

We get then

$$
\left\{\begin{array}{l}
\dot{v}_{1}=-M_{1}^{-1} K_{1} v_{1}-M_{1}^{-1} K_{2} v_{2}+M_{1}^{-1} u_{1}+\xi \cdot M_{1}^{-1} R_{D S o n} u_{2} \\
\dot{v}_{2}=-M_{2}^{-1} K_{3} v_{1}+M_{2}^{-1} u_{2}
\end{array}\right.
$$

which gives us the state space representations of the electric part of the model 


$$
\dot{X}=A X+B u
$$

The matrices are as follows

$$
\begin{aligned}
& M_{1}=\left[\begin{array}{ccc}
L_{G} & 0 & L_{S} \\
-L_{G} & L_{D} & 0 \\
-1 & -1 & 1
\end{array}\right], K_{1}=\left[\begin{array}{ccc}
R_{G} & 0 & R_{S} \\
-R_{G} & R_{D} & 0 \\
0 & 0 & 0
\end{array}\right] \\
& K_{2}=\left[\begin{array}{ccc}
1 & 0 & 0 \\
-1 & 0 & 0 \\
0 & 0 & 0
\end{array}\right] \text { and } u_{1}=\left[\begin{array}{c}
V_{G G} \\
V_{D D}-V_{G G} \\
0
\end{array}\right]
\end{aligned}
$$

and

$$
\begin{aligned}
M_{2} & =\left[\begin{array}{ccc}
C_{G S} & -C_{D G} & 0 \\
C_{G S} & 0 & C_{D S} \\
-1 & -1 & 1
\end{array}\right] \text { and } u_{2}=\left[\begin{array}{c}
0 \\
-I_{M} \\
0
\end{array}\right] \\
K_{3} & =\left[\begin{array}{ccc}
-1 & 0 & 0 \\
0 & 0 & -1 \\
0 & 0 & 0
\end{array}\right]
\end{aligned}
$$

Computing the inverse matrices $M_{1}^{-1}$ et $M_{2}^{-1}$, we get the A matrix

$$
A=\left[\begin{array}{cccccc}
\frac{-R_{G}\left(L_{D}+L_{S}\right)}{d_{1}} & \frac{R_{D} L_{S}}{d_{1}} & \frac{-R_{S} L_{D}}{d_{1}} & \frac{-L_{D}-L_{S}}{d 1} & 0 & 0 \\
\frac{R_{G} L_{S}}{d_{1}} & \frac{-R_{D}\left(L_{G}+L_{S}\right)}{d_{1}} & \frac{-L_{G} R_{S}}{d_{1}} & \frac{L_{S}}{d 1} & 0 & 0 \\
\frac{-R_{G} L_{D}}{d_{1}} & \frac{-R_{D} L_{G}}{d_{1}} & \frac{-R_{S}\left(L_{D}+L_{G}\right)}{d_{1}} & \frac{-L_{D}}{d_{1}} & 0 & 0 \\
\frac{d_{D S}}{d_{2}} & 0 & \frac{C_{D G}}{d_{2}} & 0 & 0 & 0 \\
\frac{-\left(C_{D S}+C_{G S}\right)}{d_{2}} & 0 & \frac{C_{G S}}{d_{2}} & 0 & 0 & 0 \\
\frac{-\frac{C_{G S}}{d_{2}}}{d_{2}} & 0 & \frac{C_{D G}+C_{G S}}{d_{2}} & 0 & 0 & 0
\end{array}\right]
$$

This state space representation offers a very bad numerical conditionning. We can remark that we have two null eigen values $\lambda_{1}=\lambda_{2}=0$. This means that there are two integrators in the process. This will integrate twice all the numerical computation errors. There are two big complex conjugate eigen values, in the A matrix which are for our case: $\lambda_{3}=\lambda_{34}^{*}=-1.1250 e+08+1.0468 e+08 i$. The last two eigen values are the bigest and the smallest ones $\lambda_{5}=-87.903 e+06$ and $\lambda_{6}=8.4371 e-09$. They have as ratio $\frac{\lambda_{1}}{\lambda_{2}}=-1.0419 e+16$ which show us that this matrix is very badly conditionned for simulation omputings.

This proves that this state space form cannot be used in simulations because of the numerical problems; this justifies the need of the NDA approach [1].

Second State Space formulation The fact we got two zero eigen values means that special relations exist between the state variables considered and inputs. Effectively we can remark that in the system equations we have Algebraic equations mixed with differential equations. The previous system 4 can then be rewretten to emphasize the Differential and Algebraic Equations (DAE). We can observe, in the oridnary differential equations (ODEs) of 16 and 17, that 
dependence of the three currents and the three voltages is obvious. Each one of those systems has then only 2 independent ODEs, as their third one expresses only the difference beetwen the previous ones.

$$
\begin{gathered}
\left\{\begin{array}{c}
L_{G} \frac{d i_{G}}{d t}+L_{S} \frac{d i_{S}}{d t}+R_{G} i_{G}+R_{S} i_{S}+V_{G S}=V_{G G} \\
L_{D} \frac{d i_{D}}{d t}+R_{D} i_{D}+V_{D S}+L_{S} \frac{d i_{S}}{d t}+R_{S} i_{S}=V_{D D} \\
i_{D}=i_{S}-i_{G}=i_{D G}+i_{D S}+\xi . I_{M}
\end{array}\right. \\
\left\{\begin{array}{c}
-C_{D G} \frac{d V_{D G}}{d t}+C_{G S} \frac{d V_{G S}}{d t}=i_{G} \\
C_{D S} \frac{d V_{D S}}{d t}+C_{G S} \frac{d V_{G S}}{d t}=i_{S}-\xi . I_{M} \\
V_{D S}=V_{D G}+V_{G S}
\end{array}\right.
\end{gathered}
$$

So we can remark that the variables $i_{D}$ and $V_{D S}$ (respectively) can be deduced by algebraic equations from the state variables $v_{1}=\left[i_{G}, i_{S}\right]^{T}$ and $v_{2}=\left[V_{G S}, V_{D G}\right]^{T}$ (respectively). In addition these equations include the system non linearities and thermal dependances and variations. We can replace $i_{D}=$ $i_{S}-i_{G}=i_{D G}+i_{D S}+I_{M}$. The same can be done for the voltage $V_{D S}=V_{D G}+V_{G S}$ which imposes the inherent EKV current sources. Then we get the following ODE systems become

$$
\begin{gathered}
\left\{\begin{array}{c}
L_{G} \frac{d i_{G}}{d t}+L_{S} \frac{d i_{S}}{d t}+R_{G} i_{G}+R_{S} i_{S}+V_{G S}=V_{G G} \\
-L_{D} \frac{d i_{G}}{d t}+\left(L_{D}+L_{S}\right) \frac{d i_{S}}{d t}-R_{D} i_{G}+\left(R_{D}+R_{S}\right) i_{S}+\xi \cdot R_{D S o n} I_{M}=V_{D D}
\end{array}\right. \\
\left\{\begin{array}{c}
C_{G S} \frac{d V_{G S}}{d t}-C_{D G} \frac{d V_{D G}}{d t}-i_{G}=0 \\
\left(C_{G S}+C_{D S}\right) \frac{d V_{G S}}{d t}+C_{D S} \frac{d V_{D G}}{d t}-i_{S}=-\xi . I_{M}
\end{array}\right.
\end{gathered}
$$

Finally

$$
\left\{\begin{array}{l}
\dot{v}_{1}=-M_{1}^{-1} K_{1} v_{1}-M_{1}^{-1} K_{2} v_{2}+M_{1}^{-1} u_{1} \\
\dot{v}_{2}=-M_{2}^{-1} K_{3} v_{1}+\xi \cdot M_{2}^{-1} u_{2}
\end{array}\right.
$$

The matrices are as follows

$$
\begin{aligned}
& M_{1}=\left[\begin{array}{cc}
L_{G} & L_{S} \\
-L_{D} & L_{D}+L_{S}
\end{array}\right], K_{1}=\left[\begin{array}{cc}
R_{G} & R_{S} \\
-R_{D} & R_{D}+R_{S}
\end{array}\right] \\
& K_{2}=\left[\begin{array}{ll}
1 & 0 \\
0 & 0
\end{array}\right] \text { and } u_{1}=\left[\begin{array}{l}
V_{G G} \\
V_{D D}
\end{array}\right]
\end{aligned}
$$

and

$$
\begin{aligned}
M_{2} & =\left[\begin{array}{cc}
C_{G S} & -C_{D G} \\
C_{G S}+C_{D S} & C_{D S}
\end{array}\right] \text { and } u_{2}=\left[\begin{array}{c}
0 \\
-I_{M}
\end{array}\right] \\
K_{3} & =\left[\begin{array}{cc}
-1 & 0 \\
0 & -1
\end{array}\right]
\end{aligned}
$$

The matrices determinants are $d_{1}=2.7900 .10^{-16}$ and $d_{2}=2.1135 .10^{-19}$ (respectively for $M_{1}$ and $M_{2}$ ) which means than we have interest to simulate the 
two equations separately (one for $v_{1}$ and the second for $v_{2}$ ). The system has as eigen values $\lambda_{p}$ :

$$
\lambda_{p}=10^{8}[-0.4679+6.3800 i ;-0.4679-6.3800 i ;-1.0967+1.7151 i ;-1.0967-1.7151 i]
$$

\section{Simulations Tests and Model Validation}

In order to verify the afore-mentioned characteristics, a series of simulation circuits were developed. The developed model has been implemented in Saber, Pspice and Psim softwares and compared to the experimental curves presented by the SiC MOSFET data sheet for its validation and parameters extraction.

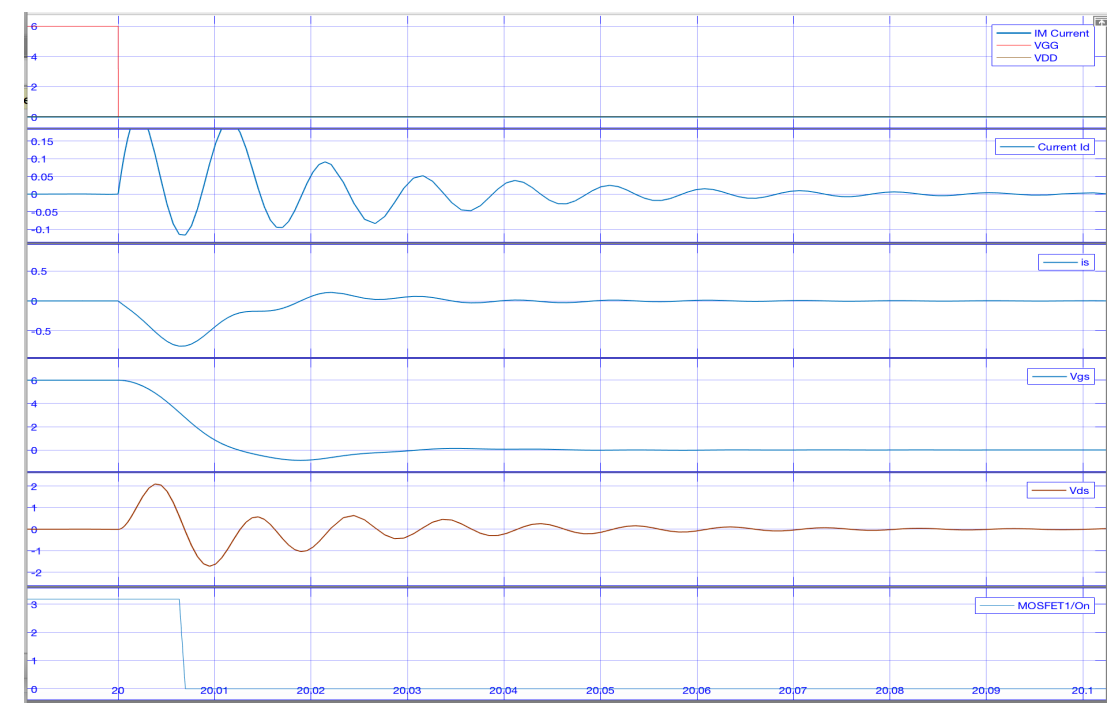

Fig. 2. Transeint responses

\section{Conclusion}

A new state space model has been proposed in this paper for a SiC Power MOSFET. It is deduced from an electric equivalent circuit, by selecting appropriate nonlinear differential equations written in a state space form.

This model can be implemented under Matlab/Simulink software and tested in simulation. The Characteristics of the MOSFET simulation models (on-state resistance, threshold voltage, and transconductance, etc...) are compared to Pspice simulation model

The simulation model of SiC power MOSFET is easy to use in numerical design and prototyping of electric circuits and simulations. The future work of our staff will be on nonlinear and robust control of a power circuit including the SiC MOSFETs. 
Acknowledgments This research activity was held by the SASV group of the LIS and co funded by an FUI project. The authors would like to thanks also Europractice (http://www.europractice.stfc.ac.uk/ ).

\section{References}

1. A. Karvonen and T. Thiringer, "Mosfet modeling adapted for switched applications using a state-space approach and internal capacitance characterization," in International Conference on Power Electronics and Drive Systems (PEDS) pp1107 - 1112 Taipei, Taiwan 2-5 Nov. 2009, 2009. 1, 2.1, 2.3, 2.3

2. A. Karvonen, "Emi from switched converters : Simulation methods and reduction techniques," Chalmers University, Licentiate Thesis at Department of Energy and Environment, Division of Electric Power Engineering; Chalmers University of Technology Goteborg, Sweden 2011, Goteborg, Sweden, 2009. 1, 2.1

3. H. Chung-Wen, A. Ruehli, and P. Brennan, "The modified nodal approach to network analysis," IEEE Transactions on Circuits and Systems, vol. 22, no. 6, pp. $504-509,1975.1,2.2$

4. L. M. Wedepohl and L. Jackson, "Modified nodal analysis: an essential addition to electrical circuit theory and analysis," Journal of Engineering Science and Education, vol. 11, no. 3, pp. 84-92, 2002. 1, 2.2

5. N. K. M'Sirdi, E. Baghaz, K. Frifita, A. Naamane, and M. BOUSSAK, "Simulation model of a sic power mosfet variables estimations and control of a power source," in 14th International Conference on Informatics in Control, Automation and Robotics - Volume 1: ICINCO, O. G. 1 and K. Madani, Eds., vol. 1, july 2017, pp. 637-643. $1,2.2,2.2$

6. K. Frifita, N. K. M'Sirdi, E. Baghaz, A. Naamane, and M. Boussak, "Electro-thermal model of a silicon carbide power mosfet," submitted to ICEERE2018, Saidia Oujda Morroco, April 2018. 1, 2.2

7. E. Vittoz, C. C. Enz, and F. Krummenacher, "A thermal characterization of power transistors semiconductor," vol. 11. 1, 2.1

8. CREE, "Sic power mosfet cmf20120d datas-sheet and application note," http://www. cree. com/products/pdf/CMF20120D. pdf, 2015. 1, 2.1

9. A. Maxim and G. Maxim, "A high accuracy power MOSFET SPICE behavioral macromodel including the device selfheating and safe operating area simulation." 2.1

10. G. Verneau, L. Aubard, J. Crébier, and J. Schanen, "Empirical power mosfet modeling: Practical characterization and simulation implantation," in 37th IAS Annual Meeting. Conference Record of theVolume4, . t. I. A. M. ConferenceIndustry Applications Conference, Ed., vol. 4, no. DOI10.1109/IAS.2002.1042785. IAS Annual Meeting (IEEE Industry Applications Society) 4:2425 - 2432, February 2002, pp. 2425-2432. 2.1 